\title{
315 CORPORA LUTEA MORPHOLOGICAL AND ECHOTEXTURAL ATTRIBUTES RELATED TO PLASMA PROGESTERONE CONCENTRATION IN HEIFERS
}

\author{
L. G. B. Siqueira, J. H. M. Viana, C. A. A. Torres, E. D. Souza, L. S. Amorim, J. F. Fonseca, L. S. A. \\ Camargo and P. L. J. Monteiro Jr
}

Reproduction, Fertility and Development 20(1) 237 - 238

\begin{abstract}
It has been suggested that ultrasound image attributes are a potential indicator of the physiological and functional status of the corpus luteum (CL). The aim of this study was to evaluate corpus luteum morphological and echotextural changes, and to correlate these changes with plasma progesterone concentration [P4] throughout the bovine estrous cycle. Crossbred heifers were scanned daily, using a Bmode, real-time ultrasound machine equipped with a 5-MHz linear-array rectal transducer, throughout a natural estrous cycle (Experiment 1; $\mathrm{n}=12$ ) or during a shorter estrous cycle, interrupted on the 10th day, by luteolysis induction (Experiment 2; $\mathrm{n}=6$ ). Blood samples were collected for further plasma [P4] analyses by RIA. Corpora lutea areas (cm2) were measured, and daily images of each CL were videotaped (VHS tapes) until digitized. Computer-assisted analyses of image attributes were performed using a custom-developed software. Daily values of luteal area, echotexture, and plasma [P4] values were analyzed by ANOVA with Tukey's test to determine differences among means of each cycle day. Pearson's correlation coefficients were calculated between luteal area, mean pixel value, pixel heterogeneity, and plasma [P4]. In the first experiment, luteal tissue area increased to a maximum on the 10th day $(\mathrm{P}<0.05)$, followed by a plateau, and then declined from Day 14 to next estrus. There was a significant correlation between luteal tissue area and plasma $\mathrm{P} 4(\mathrm{r}=0.69 ; \mathrm{P}<0.01)$. In the second experiment, plasma $\mathrm{P} 4$ dropped to basal values $24 \mathrm{~h}$ after luteolysis induction. Luteal tissue area decreased at a slow rate, and reached values similar to ones from metestrus $36 \mathrm{~h}$ after treatment. In Experiment 1, echotexture parameters of the CL were analyzed after data adjustment to the onset of luteolysis. In both experiments, mean pixel values did not change throughout the estrous cycle and there was no correlation between mean pixel values and plasma $[\mathrm{P} 4](\mathrm{P}>0.10)$. Pixel heterogeneity changed throughout the natural estrous cycle, with maximum value on metestrus (Day 14; Day $0=$ luteolysis) and minimum on diestrus (Day 2; $\mathrm{P}<0.01$ ). However, this parameter did not change when luteolysis was induced (Experiment 2; $\mathrm{P}>0.10$ ). There were significant correlations between pixel heterogeneity and plasma progesterone in both of the experiments $(r=-0.69$ and $r=-0.48 ; P<0.05)$. In conclusion, mean pixel values do not reflect morphological or functional changes of the CL throughout the estrous cycle. On the other hand, based on the correlations between pixel heterogeneity and systemic [P4] in both experiments, this image attribute (heterogeneity) has the potential to indicate functionality and steroidogenic capacity of the luteal gland.
\end{abstract}

Full text doi:10.1071/RDv20n1Ab315

(C) CSIRO 2008 